\title{
Inhibition of JMJD6 expression reduces the proliferation, migration and invasion of neuroglioma stem cells
}

\author{
D. X. ZHOU, D. ZHOU, S. Q. ZHAN, P. WANG, K. QIN, W. GAN, X. F. LIN* \\ Department of Neurosurgery, Guangdong General Hospital, Guangzhou 510080, China \\ *Correspondence: xflin9666@sina.com
}

Received November 28, 2016 / Accepted February 8, 2017

\begin{abstract}
Neuroglioma is the most common form of human primary malignant brain tumor, more and more studies recently showed only a small subpopulation of glioma cells which called glioma stem cells have true tumorigenic potential. Meanwhile, it was reported the overexpression of JMJD6 protein is closely involvement with the occurrence and development of multiple tumors, and JMJD6 is required for the differentiation of multiple organ, tissues and cells during embryogenesis. However, the influence of JMJD6 overexpression on neuroglioma development is unclear now. Hence, to explore the effects of JMJD6 expression on neuroglioma, we firstly isolated glioma stem cells by using CD133 MicroBead Kit, and identified via neurosphere-forming assay and Immunofluorescence staining. At the same time, we investigated the effects and mechanism of JMJD6 on the proliferation, migration and invasion of glioma stem cells through MTT, transwell assays and the Cignal finder cancer 10-pathway reporter array. The results demonstrated that the glioma neurosphere cells positively expressed stem cell marker SOX2, neuroectodermal stem cell marker Nestin, and also expressed astrocytes marker GFAP and neurons marker $\beta$-tubulin III fter FBS-induced differentiation for a week, which proved the glioma neurosphere cells have the selfrenewal and multipotential differentiation capacity. Moreover, shRNA lentiviral vector mediated knockdown of JMJD6 in glioma stem cells led to decreased proliferation, migration and invasion, the underlying molecular mechanism is related to the weaken of Wnt signaling pathway and strengthen of p53 signaling pathway.
\end{abstract}

Key words: JMJD6, neuroglioma, neuroglioma stem cell

Neuroglioma, also called gliocytoma, is the most common form of human primary malignant brain tumor at present. It occupies more than $50 \%$ of brain cancers, with about three new cases per 100,000 populations per year $[1,2]$, and is characterized with high invasion, morbidity and mortality, and endangers human life and health seriously $[3,4]$. In the neuroglioma patients, fewer than $10 \%$ of them could survive beyond 5 years [5]. The neuroglioma pathogenesis is related to multiple processes as affected by dozens of regulatory factors [6]. Up to now, our knowledge about the potential underlying factors regulating neuroglioma progression is still not entirely clear.

Jumonji domain-containing 6 (JMJD6) is a member of the Jumonji $\mathrm{C}$ domain-containing family of proteins. It was first considered as a phosphatidylserine receptor on cell membrane mediating phagocytosis in apoptotic cells [7]. Subsequently, various studies showed that JMJD6 has a catalytic activity as dioxygenase in the nucleus and is not involved in apop- totic cell clearance $[8,9]$. Now, JMJD6 is mainly described as a protein with bifunctional histone arginine demethylation and lysyl-5-hydroxylase activity [10], although the potential functional importance of these activities remains unclear. Recently, more and more studies showed that over-expression of JMJD6 protein is strongly involved with poor prognosis in multiple cancers, such as breast cancer, lung cancer, Colon cancer, et al $[8,10,11]$. It promotes the cellular proliferation and invasion, and plays an oncogene-like role in the development of these cancers. Moreover, It was reported that the function of hydroxylating RNA splicing factors mediated by JMJD6 is essential for the differentiation of multiple tissues and cells during embryogenesis [12]. And silencing of JMJD6 weakens angiogenic functions of endothelial cells via regulating the gene expression and the splicing of the VEGF receptor 1 (Flt1) [13].

All the evidence above showed JMJD6 participates the differentiation of stem cells and exerts an important role in 
tumor biological functions. However, the role of JMJD6 in neuroglioma is still unclear. Hence, in this study, we sought to clarify the molecular mechanism in the occurrence, development of neuroglioma by investigating the functions of JMJD6 in neuroglioma stem cells, and provide the new theoretical basis for the treatment of neuroglioma in clinical.

\section{Materials and methods}

Material and reagent. Glioma cells U87 and U251 were purchased from ATCC. FBS was purchased from Gibco. Dulbecco's modified eagle medium was purchased from Hyclone. CD133 MicroBead Kit was purchased from Miltenyi Biotec. EGF, bFGF and B27 were purchased from Gibco. Anti-SOX2 were purchased from Cell Signaling Technology. Anti-Nestin, anti-JMJD6, anti-GAPDH, anti-p53, anti-TCF7L2, antiGFAP and anti- $\beta$-tubulin III, Anti-Mouse IgG H\&L (FITC), Anti-Rabbit IgG H\&L (FITC), Anti- Mouse IgG H\&L (Alexa Fluor ${ }^{\circledR}$ 647) and Anti-Rabbit IgG H\&L (Alexa Fluor ${ }^{\circledR}$ 647) were purchased from Abcam. Goat anti-rabbit or goat anti-mouse IgG-HRP was purchased from Beyotime. Lipofectamine 2000 was purchased from Invitrogen. Cignal Finder Cancer 10-Pathway Reporter Array CCA-101L was purchased from QIAGEN.

Culture and isolation of glioma stem cells. U87 and U251 cells were cultured with complete DMEM containing $10 \%$ FBS, $100 \mathrm{U} / \mathrm{mL}$ penicillin, and $100 \mu \mathrm{g} / \mathrm{mL}$ streptomycin in $5 \% \mathrm{CO}_{2}$ at $37^{\circ} \mathrm{C}$. When glioma cell confluence reached $80 \%$, cells were harvested by trypsinization collected by centrifugation and washed with PBS for 3 times. Then the cells were incubated with mAbs against CD133-PE and CD133 MicroBeads according to the instruction manual. Finally, Glioma stem cells (CD133+ cells) were isolated from the tubular fraction by magnetic sorting, using the MACS system.

Sphere-forming assay. Glioma stem cells (1000 cells per well) were plated onto a 24-well ultra-low attachment plate and cultured in serum-free DMEM/F12 medium supplemented with $20 \mathrm{ng} / \mathrm{mL}$ EGF, $20 \mathrm{ng} / \mathrm{mL}$ bFGF, $2 \%$ B27 in $5 \% \mathrm{CO}_{2}$ at $37^{\circ} \mathrm{C}$. After 7 days of culture, the spheres were observed under an inverted microscope and identified by immunofluorescence staining.

Immunofluorescence staining. The U87 and U251 stem cells (U87-SC or U251-SC) were seeded onto a 24-well plate and fixed in $4 \%$ paraformaldehyde for $20 \mathrm{~min}$, permabilized with $0.1 \%$ Triton X-100 and subsequently blocked with $10 \%$ donkey serum for $1 \mathrm{~h}$. Then the cells were incubated at $4{ }^{\circ} \mathrm{C}$ overnight with one of the following antibodies: mouse antiNestin (1:1000), rabbit anti-SOX2 (1:400), rabbit anti-GFAP $(1: 1000)$ and mouse anti- $\beta$-tubulin III $(1: 1000)$. Next day, cells were washed with PBS for 3 times and incubated with corresponding secondary antibodies including Alexa Fluor 647-conjugated goat anti-mouse or goat anti-rabbit (1:500) and FITC-conjugated goat anti-rabbit or goat anti-mouse (1:1000). Nuclei were counterstained with DAPI and imaged under a fluorescence microscope.
Transfection and western blot. Glioma stem cells were divided into shRNA-NC group and shRNA-JMJD6 group. JMJD6-shRNA lentiviral vector and vector control were constructed in FulenGen (Guangzhou, China). Cells were harvested using trypsin and lysed with RIPA buffer, the supernatants were collected by centrifugation. Then the protein was denatured by boiling and detected the concentration by BCA Protein Assay reagent kit. The protein was fractionated using SDS-PAGE and transferred onto PVDF membrane, and membranes were blocked for $1 \mathrm{~h}$ in $5 \%$ skim milk before being incubated with antibodies. For detection, the membrane were incubated at $4{ }^{\circ} \mathrm{C}$ overnight with one of the following antibodies: rabbit anti-JMJD6 (1:500), mouse anti-GAPDH (1:5000), rabbit anti-p53 (1:1000), rabbit antiTCF7L2 (1:25000), and washed 3 times in TBST, each time for $10 \mathrm{~min}$. Then the membrane was incubated with secondary antibodies (goat anti-mouse or goat anti-Rabbit IgG H\&L) (1:1000) for $1 \mathrm{~h}$ at room temperature. Finally, the membrane was washed with TBST repeatedly again and the protein was visualized using the ECL kit and observed by GeneGnome mechine (Syngene).

MTT assay. Cell proliferation was evaluated after transfected with JMJD6-shRNA lentiviral vector. Firstly, cells in the logarithmic growth phase were harvested by trypsin and centrifugation, and resuspended. Then $100 \mu \mathrm{L}$ suspension containing 1000 cells were plated into 96-well plates per well and cells were grown for $24 \sim 72 \mathrm{~h}$. Four parallel wells were designed for each experimental group. Then $20 \mu \mathrm{L}$ MTT $(5 \mathrm{mg} / \mathrm{ml})$ was added to each well and incubated for $4 \mathrm{~h}$. Finally, the reaction was terminated by $150 \mu \mathrm{L}$ DMSO. And the optical density at $490 \mathrm{~nm}$ was detected using Microplate Reader.

Cell migration and invasion assay. $200 \mu \mathrm{L}$ Serum-free DMEM containing $1.5 \times 10^{5}$ U87-SC or U251-SC were placed into the upper room of a Transwell insert, while the lower room was filled with completed DMEM containing 10\% FBS as a chemoattractant. The filter of Transwell insert without Matrigel were used for transwell migration assay and coated with Matrigel to act as extracellular matrix were used for invasion assay. Then the Transwell system was incubated at $37^{\circ} \mathrm{C}$ for $24 \mathrm{~h}$. And migrated and invaded cells were fixed with methanol for $20 \mathrm{~min}$, rinsed with PBS and stained with crystal violet for $15 \mathrm{~min}$. Cells were counted in four randomly selected fields under microscope.

Cignal finder cancer 10-pathway reporter array. The Cignal finder cancer 10-pathway reporter array was performed as described to pinpoint pathways which regulated the effect of JMJD6 possibly in glioma stem cells. After preparation of complex formation according to the instruction manual, the logarithmic growth phase cells were harvested by trypsin and centrifugation, and resuspended. Then $50 \mu \mathrm{L}$ suspension $\left(1 \times 10^{6} / \mathrm{ml}\right)$ were plated into 96 -well plates per well and cultured at $37^{\circ} \mathrm{C}$ with $5 \% \mathrm{CO}_{2}$ for $6 \mathrm{~h}$, and previous medium was replaced with medium which containing $10 \%$ FBS without antibiotics subsequently. Next day, complete medium were added and cells were cultured continuously for $48 \mathrm{~h}$. Finally, 

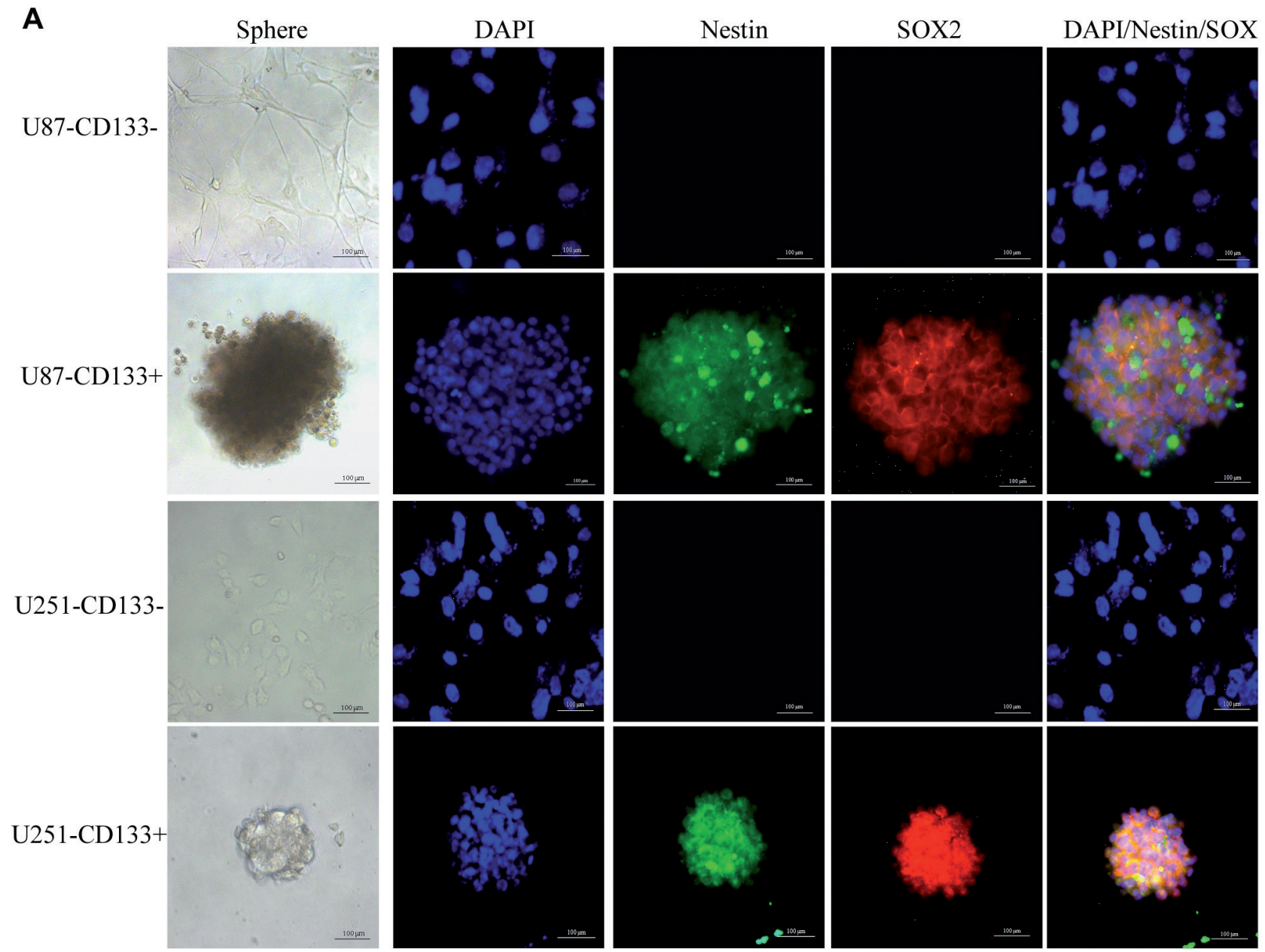

B
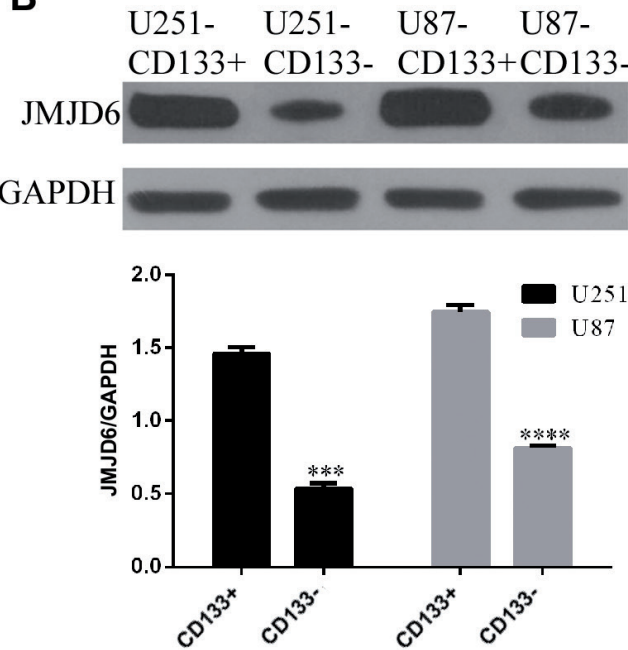

C

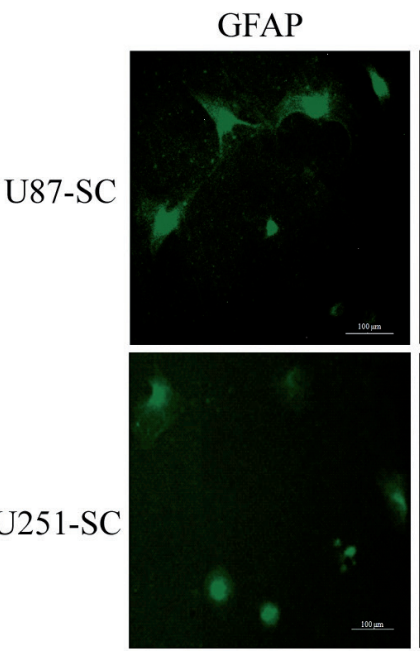

$\beta$-Tubulin $3 \quad$ GFAP $/ \beta$-Tubulin 3

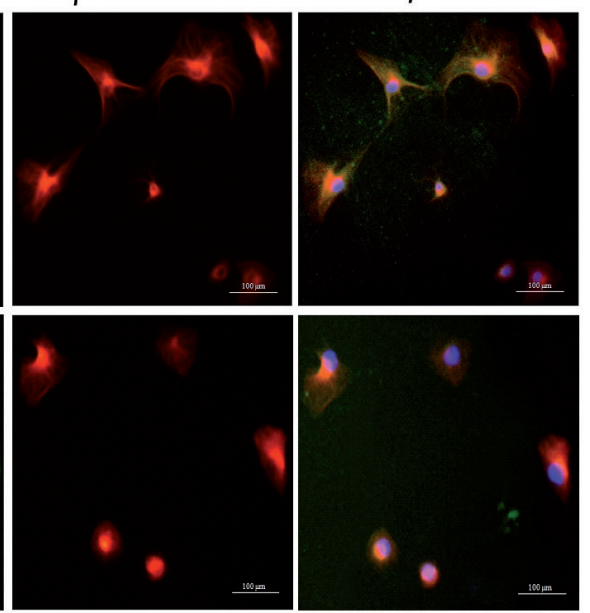

Figure 1. Isolation and identification of glioma stem cells. (A) Line1: The sphere morphology of glioma stem cells (CD133+ cells) was observed and imaged under invert microscope; Line2 Line5: Before differentiation, neurosphere was identified by immunefluorescence staining, and the stem cell marker SOX2 and neuroectodermal stem cell marker Nestin were both expressed positively in neurosphere. (B) The expression of JMJD6 were detected via Western blot. Compared with CD133- cells, the expression of JMJD6 was significantly increased in glioma stem cells (CD133+ group). CD133- group as control, compared to control group, ${ }^{* *} P<0.001 ;{ }^{* * *} P<0.0001$. (C) After FBS-induced for a week, the markers of differential neural cell lineages like GFAP and $\beta$-tubulin III were expressed in CD133+ cells. 


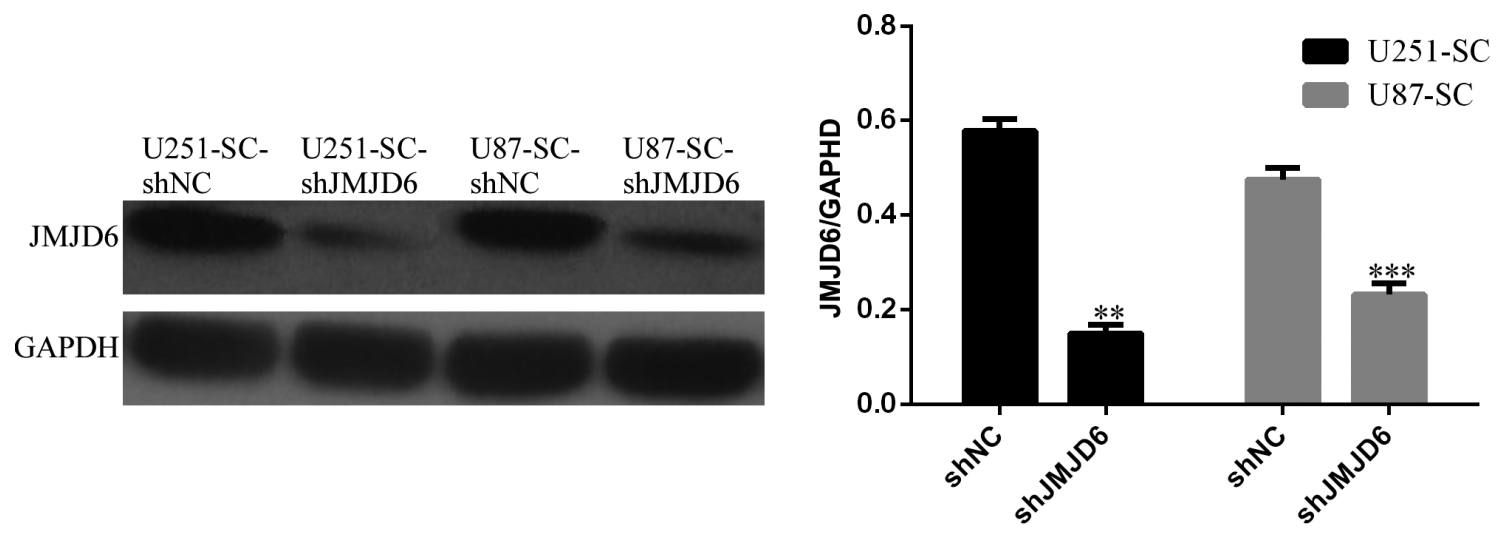

Figure 2. JMJD6 protein expression levels of Glioma stem cells (U87-SC/U251-SC). Cells transfected with shRNA-JMJD6 lentiviral vector and NC vector were considered as experimental groups and control groups, respectively. Western blot was performed to verify transfection efficacy. Left: The expression of JMJD6 was down-regulated notably in glioma stem cells after transfected with shRNA-JMJD6 lentiviral vector. Right: Relative expression of JMJD6. Compared to shNC group, ${ }^{* * P}<0.01 ;{ }^{* * * P<0.001}$.

the luciferase assay was performed using Dual-Luciferase Reporter Assay System (Promega, WI, USA).

Statistical analysis. All experiment data were expressed as mean $\pm \mathrm{SD}$, and Statistical analyses were performed using SPSS 19.0. Significance between two groups was examined with unpaired Student's $t$ test for two groups; one-way ANOVA for multiple groups. Differences were considered statistically significant at $P<0.05$.

\section{Results}

Isolation and identification of neuroglioma stem cells. In this study, we firstly isolated glioma stem cells (U87-SC and U251-SC) using CD133 MicroBead Kit. For the CD133+ cells, Sphere-forming assay was performed in a 24-well ultralow attachment. The result showed CD133+ cells have the neurosphere-forming ability. The sphere morphology was formed after cultured for a week, and imaged under an invert microscope (Figure 1A, Line1).

In order to identify whether the CD133+ cells were neuroglioma stem cells, immunofluorescence staining was used to detect the expression of stem cell specific markers. As shown in Figure 1A (Line2 Line5), stem cell marker SOX2 and neuroectodermal stem cell marker Nestin were both expressed positively in U87-SC and U251-SC. Moreover, after FBS-induced differentiation for a week, we detected the markers of different neural cell lineages including astrocytes (GFAP) and neurons ( $\beta$-tubulin III) were also expressed positively (Figure 1C), all evidences above suggested the glioma neurosphere cells we isolated had the capacities of self-renew and multipotential differentiation.

The expression of JMJD6 in neuroglioma stem cells. The expression of JMJD6 protein was analyzed by Western blot in CD133- and CD133+ glioma cells (U87 and U251), respectively. Compared with CD133-glioma cells, JMJD6 expression increased notably in CD133+ glioma cells $(P<0.001$, Figure 1B).
Lentiviral vector infection and JMJD6 silencing. After understanding the difference of JMJD6 expression between CD133- and CD133+ glioma cells, the silencing efficacy of JMJD6 in CD133+ glioma cells (neuroglioma stem cells) was determined by Western blot, and the results showed the expression level of JMJD6 decreased dramatically compared with that in negative control groups $(P<0.01$, Figure 2$)$.

JMJD6 promotes neuroglioma stem cell proliferation. The effect of JMJD6 on neuroglioma stem cell proliferation was assessed via MTT assay. And results showed cell viability decreased significantly in U87-SC and U251-SC after transfected with JMJD6-shRNA lentiviral vector $(P<0.05$ or $P<0.01$; Figure 3). That is to say JMJD6 could promote the proliferation of neuroglioma stem cell.

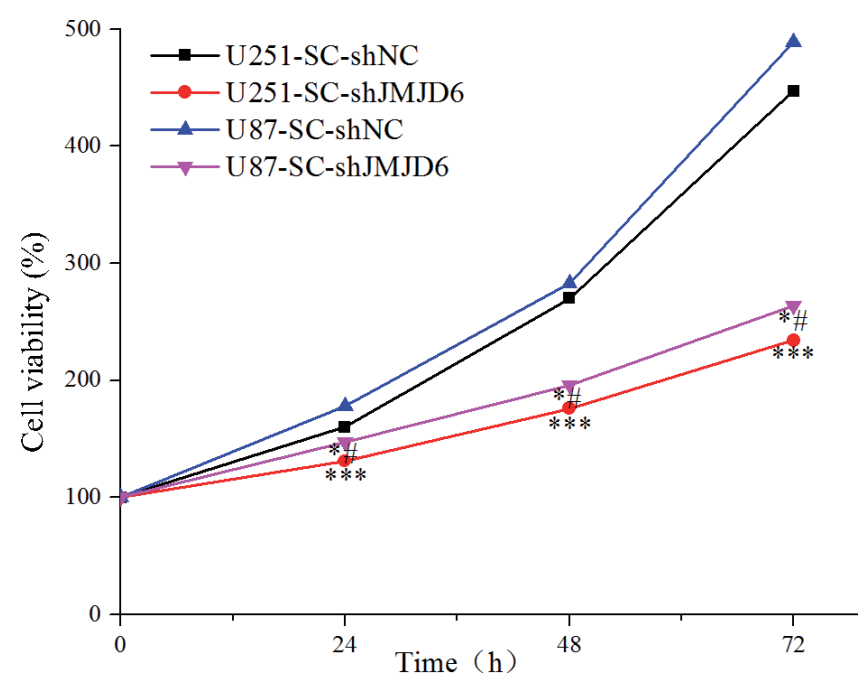

Figure 3. MTT assay was used to detected cell viability. The proliferation ability of glioma stem cells reduced after JMJD6-shRNA lentiviral vector transfecion. Compared with shNC group, ${ }^{* * * P}<0.001 ;{ }^{\star} \# P<0.01$. 
JMJD6 accelerates neuroglioma stem cell migration and invasion. Transwell migration and matrix penetration assays were used to examine the effect of JMJD6 on migratory and invasive abilities. Compared to control group, the migration speed was reduced in neuroglioma stem cells (U87-SC and U251-SC) after transfected with JMJD6-shRNA lentiviral vector $(P<0.01$, Figure $4 \mathrm{~A})$. Moreover, cell invasion ability was reduced as well after JMJD6-shRNA lentiviral vector transfection $(P<0.01$, Figure $4 \mathrm{~B})$. In brief, JMJD6 could promote migration and invasion of neuroglioma stem cells.
JMJD6 exerts its function by activating Wnt signaling and suppressing P53 signaling. To investigate the mechanisms by which JMJD6 promotes glioma stem cell proliferation, migration and invasion, we firstly adopted the Cignal finder cancer 10-pathway reporter array to explore signaling pathways involved in these processes. The results showed that Wnt signaling was suppressed and p53/DNA damage signaling was activated in neuroglioma stem cells after transfected with JMJD6-shRNA lentiviral vector (Figure 5A, 5B). To verify the result above, we measured the expression levels of TCF7L2 and p53, which is the
A

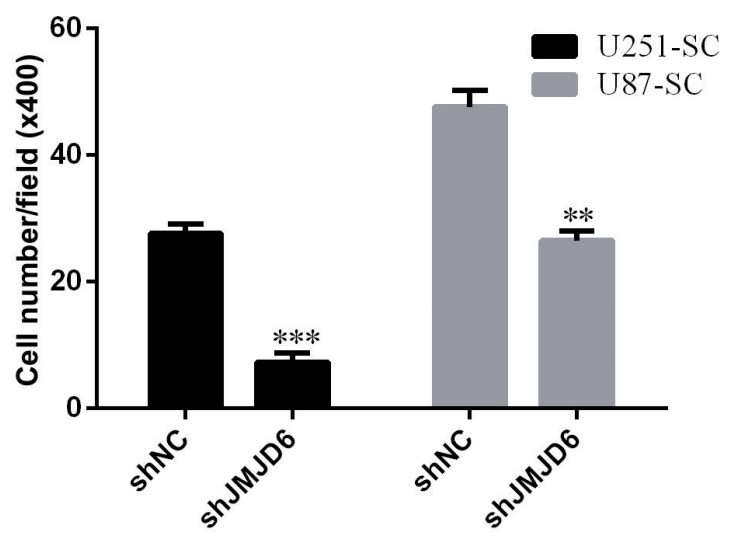

B

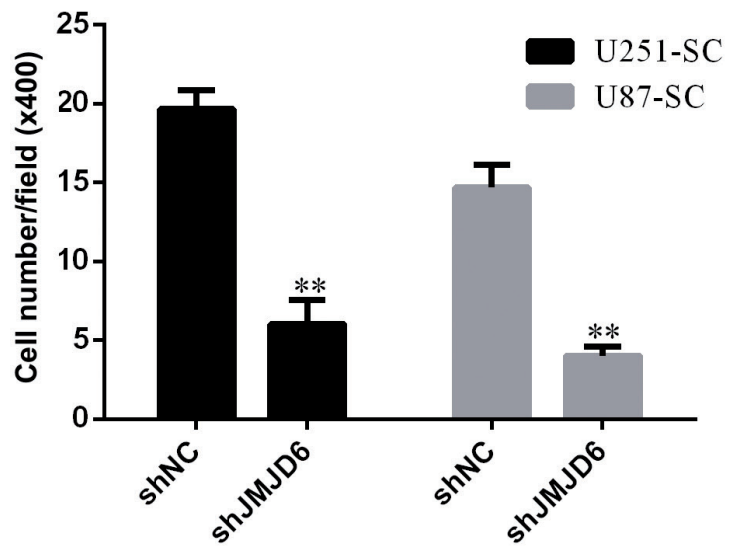

U251-SC-shNC

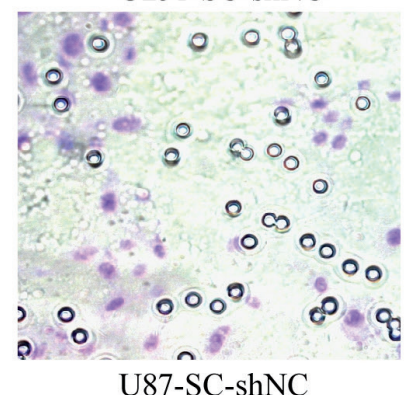

U251-SC-shJMJD6

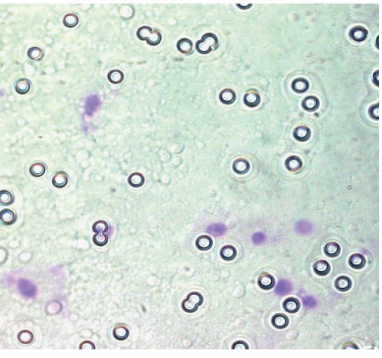

U87-SC-shJMJD6
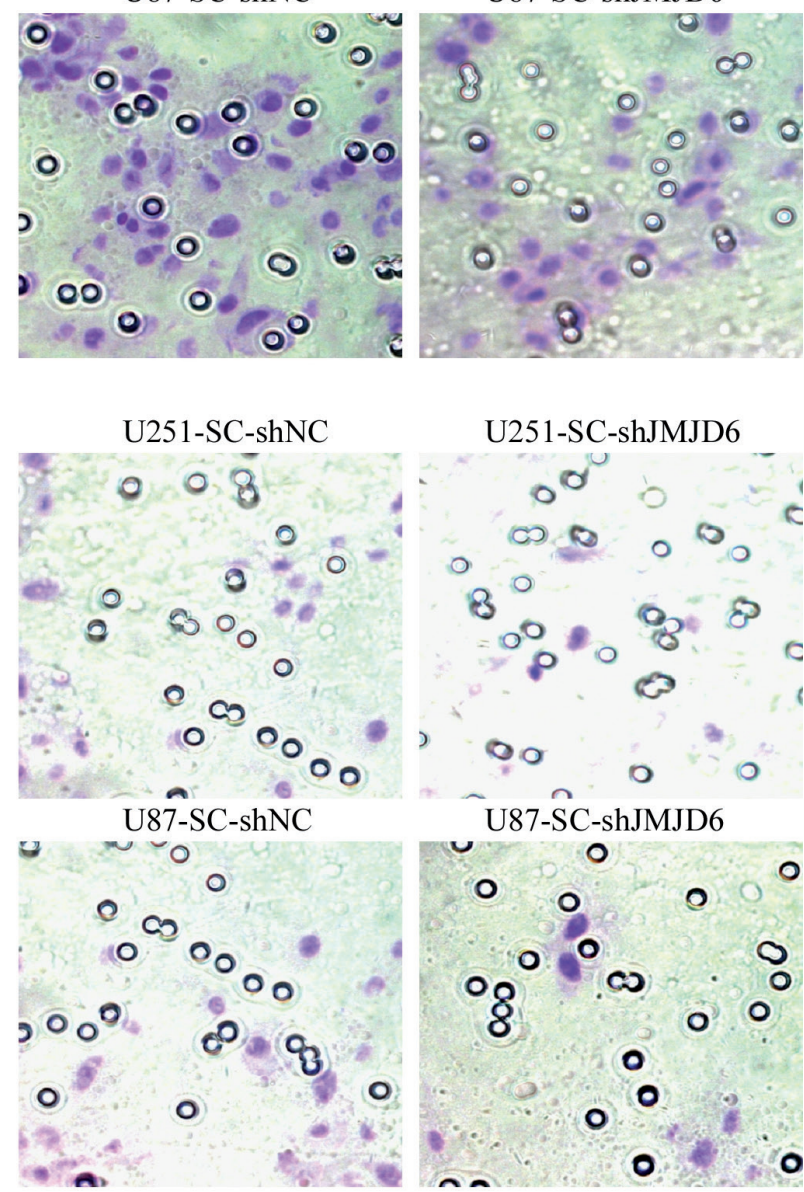

Figure 4. Inhibition of JMJD6 could reduce migration and invasion ability of glioma stem cells (U87-SC and U251-SC). (A) Cell migration; (B) Cell invasion. U87-SC-shNC/ U251-SC-shNC as control groups. Compared with control group, ${ }^{* *} P<0.01 ;{ }^{* *} P<0.001$. 
important molecule in Wnt and p53/DNA damage signaling pathway, respectively. And results indicated that silencing of JMJD6 decreased the expression of TCF7L2 and increased the expression of p53 notably in JMJD6-shRNA lentiviral transfection groups (Figure 6A, 6B).

\section{Discussion}

Neuroglioma is the most frequent malignant primitive tumors of central nervous system, and statistical data show that the annual morbidity is about $10-20 / 100,000$ people. This disease has a wide range of onset age, it occurs to people in age of 20-50 years old with 5-year survival rate lower than 5\% [14]. Currently, despite great advances in therapy, none of the treatments is curative. Previous studies show that the malignant degree has a positive correlation with the ability cytotoxin resistance, invasion transfer and proliferation which induced by tumor vessel, the main reason is neuroglioma always presents invasive growth and has no obvious demarcation in normal brain tissues [15]. The pathogenesis of neuroglioma is complex and involves with dysregulation of many biological pathways at multiple levels [16], and despite the main view which caused neuroglioma is due to the interaction between genetic factors and environmental factors, the specific mechanism is not yet clear [17-19]. Hence, it is important to deeply understand and research the occurrence and development mechanism of neuroglioma.

Previous studies show heterogeneous cell populations exist in glioma, these different subpopulation cells are result due to the loss of genomic stability, or due to the dysregulated proliferation and differentiation processed in glioma cells, and the subpopulations exhibit distinct abilities regarding their contribution to glioma growth and development [20]. A small part of glioma subpopulation cells which characterized with tumor-initiating/stem cells function are defined as glioma initiating/stem cells (GICs/GSCs) [4]. Interesting, it was reported that glioma stem cells are shown to have significant similarities to normal neural stem cells (NSCs), including the self-renew ability, similar transcriptome profiles, and ability differentiation into different neuronal lines [21]. The self-renew and differentiation capacity of normal neural stem cells can be evaluated via surrogate neurosphere-forming assay [22-26]. Therefore, in this study, we isolated glioma stem cells from glioma cells by using CD133 MicroBead Kit, then identified via neurosphere-forming assay, immunofluorescence staining and FBS-induced differentiation experiments. The result showed the glioma stem cells (U251/U87-CD133+ cells) had neurosphere-forming ability (Figure 1A, Line1) and positively expressed stem cell marker SOX2 and neuroectodermal stem cell marker Nestin (Figure 1A, Line2 Line5). Moreover, after FBS-induced differentiation for a week, the neurosphere cells expressed astrocytes marker GFAP and neurons marker $\beta$-tubulin III (Figure 1C), which suggested the glioma neurosphere cells have the self-renew and multipotential differentiation capacity.
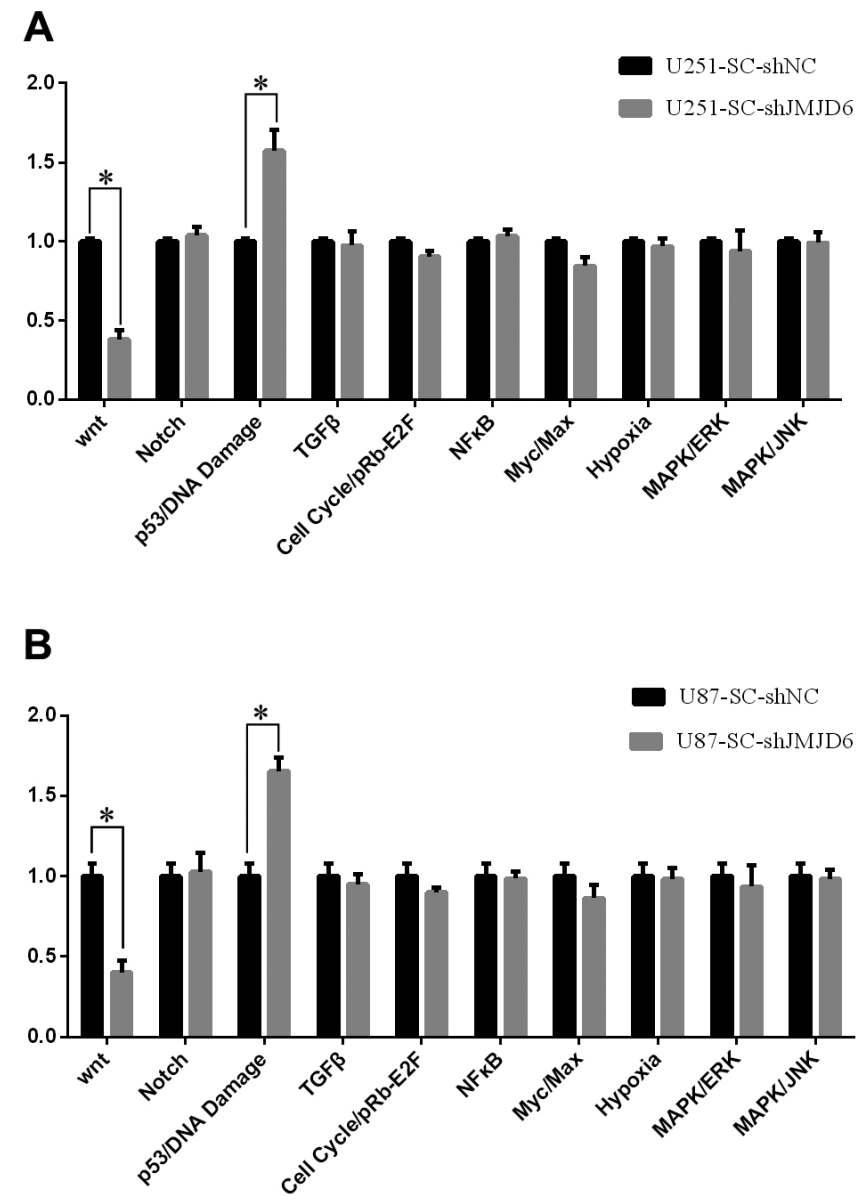

Figure 5. JMJD6 stimulates Wnt and p53/DNA damage signaling in glioma stem cells. Glioma stem cells transfected with JMJD6-shRNA or NC vectors were used in these studies. (A, B) Wnt and p53/DNA damage signaling were both stimulated in U251-SC and U87-SC, respectively, while the other signaling pathways showed no significant change.

JMJD6 protein has been identified for many years, the mainly functions are arginine demethylase and lysyl5-hydroxylase activities. A structural study had suggested that the substrates of JMJD6 might be methyl groups on single-stranded RNAs (ssRNAs) [27]. Recent years, multiple studies demonstrate that overexpression of JMJD6 is closely involvement with the occurrence and development of breast, lung cancer, colon cancer, et al $[8,11,28]$. Meanwhile, it was reported that JMJD6 is required for the differentiation of multiple organ, tissues and cells during embryogenesis [9]. However, the role of JMJD6 in neuroglioma is still unclear. Based on the functions of tumor stem cells and JMJD6, we detected the expression of JMJD6 in glioma stem cells via Western blot and found a significant difference in the expression between glioma stem cells (CD133+) and giloma cells (CD133-) (Figure 1B). Furtherly, we investigated the effects of JMJD6 on the proliferation, migration and invasion of glioma stem cells through MTT and transwell assays. The 

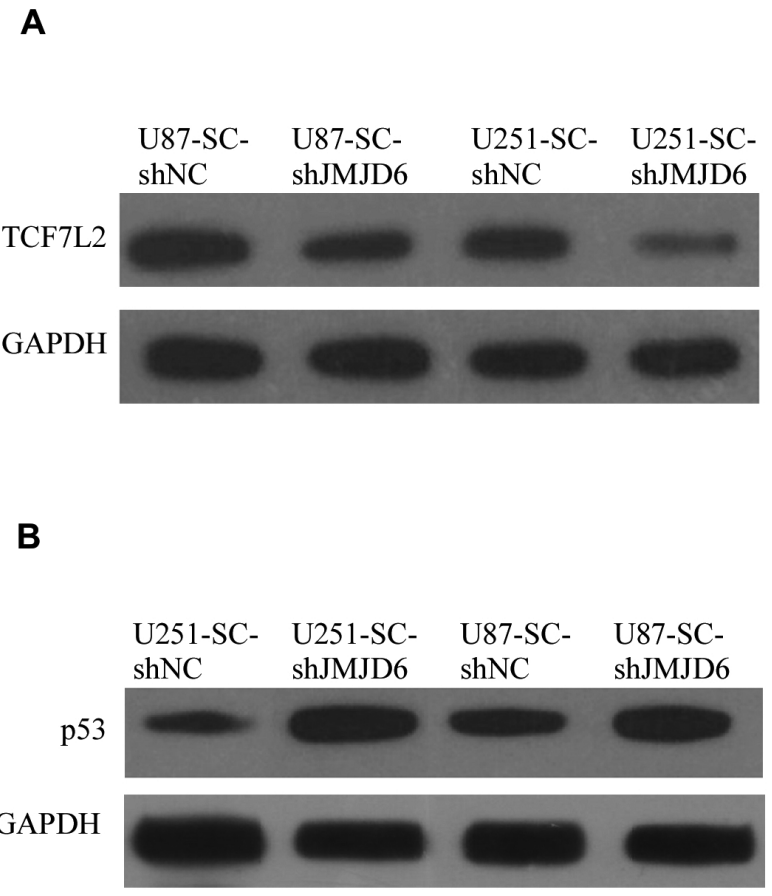
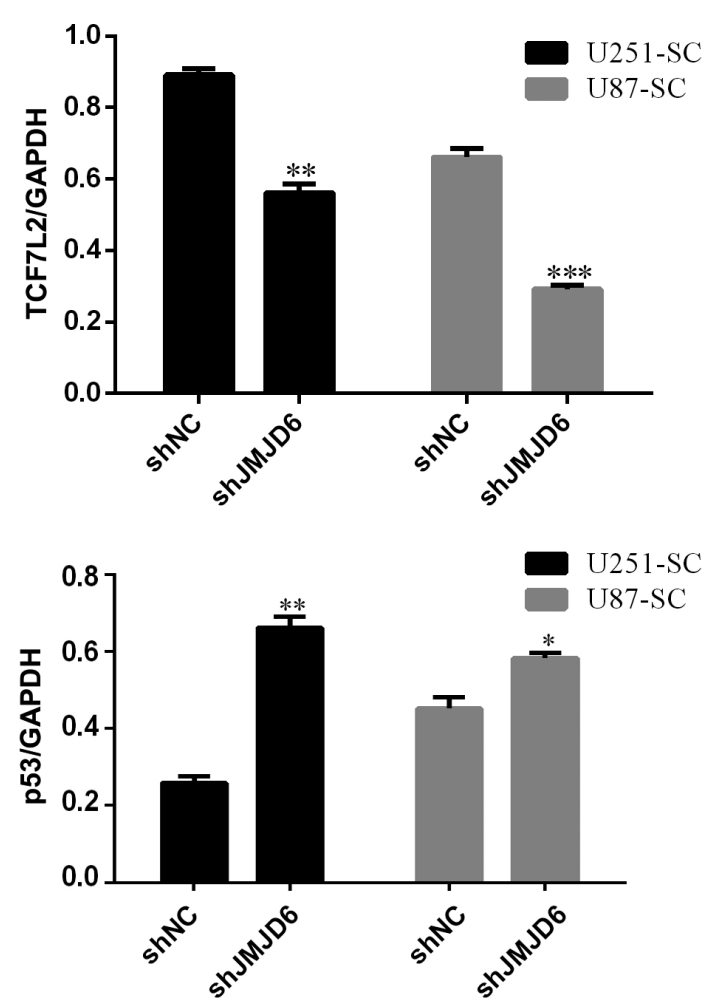

Figure 6. The expression of TCF7L2 and 533 protein levels. (A) The expression of TCF7L2, which is an important downstream molecule in Wnt signal pathway, was detected via Western blot. And TCF7L2 expression of glioma stem cells was reduced significantly after JMJD6-shRNA lentiviral vector transfection. (B) The expression of critical molecule in p53 signal pathway was increased compared to control groups. Compared with control group, ${ }^{\star} P<0.05,{ }^{*} P<0.01,{ }^{* *} P<0.001$.

results demonstrated that shRNA lentiviral vector mediated knockdown of JMJD6 in glioma stem cells led to decreased proliferation, migration and invasion (Figure 3,4). To explore the underlying mechanism, the Cignal finder cancer 10-pathway reporter array was performed to pinpoint pathways which regulated the effect of JMJD6 possibly in glioma stem cells. The results showed Wnt signaling pathway was weakened and p53 signaling pathway was enhanced in U251SC/U87-SC-shJMJD6 groups (Figure 5). As we know, p53 is a tumor suppressor, it plays a critical role in maintaining genomic stability and preventing tumor formation [29]. In previous study, Wang et al [30] has reported that JMJD6 could negatively regulate $\mathrm{p} 53$ signaling through hydroxylation which occurs on lysine 382 site of p53 protein and promote colon carcinogenesis. Meanwhile, JMJD6 antagonizes p53 acetylation and promotes the association with its negative regulator MDMX, and represses transcriptional activity of p53. And Wnt signaling also has a close relationship with tumor, its abnormal activation acts an important role in the occurrence and development of human tumors [31]. In this process, Wnt $/ \beta$-catenin signaling is mediated by protein complexes of individual members of Tcf/Lef family of DNA-binding factors [32]. Zhang et al [33] had reported that JMJD6 could enhance $\beta$-catenin-induced gene activation through antagonizes the repression effect of Tcf7ll on downstream gene transcription. In addition, in Wetering's study [34], it was reported that disruption of $\beta$-catenin/TCF-4 activity in colorectal cancer cells induces a rapid G1 arrest and controls proliferation and differentiation, inhibition the activity of $\beta$-catenin/TCF- 4 complex could block the proliferation of colorectal cancer cells effectively. All the evidence above show Wnt and p53 signaling pathways possibly make an important role in caner. Hence, investigating the correlation between JMJD6 and Wnt (or p53) signaling furtherly may provide a novel view and therapeutic target as well as a prognostic marker in neuroglioma.

In conclusion, our study above demonstrated that JMJD6 acts an important role in the processes of migration and invasion in neuroglioma, and the underlying molecular mechanism may involve Wnt and p53 signaling. However, the detailed interaction mode and processes between JMJD6 and Wnt (or p53) signaling pathway in glioma stem cells has not studied in this article and need to be investigated further.

Acknowledgments: All authors sincerely acknowledge the support given by the Guangdong General Hospital, and thank the funding support from Guangdong province science and technology plan project (NO. 2012B031800165). 


\section{References}

[1] OSTROM QT, GITTLEMAN H, FARAH P, ONDRACEK A, CHEN Y et al. CBTRUS statistical report: Primary brain and central nervous system tumors diagnosed in the United States in 2006-2010. Neuro Oncol 2013; 15 Suppl 2: ii1-56.

[2] OSTROM QT, GITTLEMAN H, FULOP J, LIU M, BLANDA R et al. CBTRUS Statistical Report: Primary Brain and Central Nervous System Tumors Diagnosed in the United States in 2008-2012. Neuro Oncol 2015; 17 Suppl 4: iv1-iv62. https:// doi.org/10.1093/neuonc/nov189

[3] MIAO H, GALE NW, GUO H, QIAN J, PETTY A et al. EphA2 promotes infiltrative invasion of glioma stem cells in vivo through cross-talk with Akt and regulates stem cell properties. Oncogene 2015; 34: 558-567. https://doi.org/10.1038/ onc. 2013.590

[4] BOURKOULA E, MANGONI D, IUS T, PUCER A, ISOLA $M$ et al. Glioma-associated stem cells: a novel class of tumor-supporting cells able to predict prognosis of human low-grade gliomas. Stem Cells 2014; 32: 1239-1253. https:// doi.org/10.1002/stem.1605

[5] STUPP R, HEGI ME, MASON WP, VAN DEN BENT MJ, TAPHOORN MJ et al. Effects of radiotherapy with concomitant and adjuvant temozolomide versus radiotherapy alone on survival in glioblastoma in a randomised phase III study: 5 -year analysis of the EORTC-NCIC trial. Lancet Oncol 2009; 10: 459-466. https://doi.org/10.1016/S1470-2045(09)70025-7

[6] PARSONS DW, JONES S, ZHANG X, LIN JC, LEARY RJ et al. An integrated genomic analysis of human glioblastoma multiforme. Science 2008; 321: 1807-1812. https://doi.org/10.1126/ science. 1164382

[7] FADOK VA, BRATTON DL, ROSE DM, PEARSON A, EZEKEWITZ RA et al. A receptor for phosphatidylserine-specific clearance of apoptotic cells. Nature 2000; 405: 85-90. https:// doi.org/10.1038/35011084

[8] WANG F, HE L, HUANGYANG P, LIANG J, SI W et al. JMJD6 promotes colon carcinogenesis through negative regulation of p53 by hydroxylation. PLoS Biol 2014; 12: e1001819. https:// doi.org/10.1371/journal.pbio.1001819

[9] BOSE J, GRUBER AD, HELMING L, SCHIEBE S, WEGENER I et al. The phosphatidylserine receptor has essential functions during embryogenesis but not in apoptotic cell removal. J Biol 2004; 3: 15. https://doi.org/10.1186/jbiol10

[10] LEE YF, MILLER LD, CHAN XB, BLACK MA, PANG B et al. JMJD6 is a driver of cellular proliferation and motility and a marker of poor prognosis in breast cancer. Breast Cancer Res 2012; 14: R85.

[11] ZHANG J, NI SS, ZHAO WL, DONG XC, WANG JL. High expression of JMJD6 predicts unfavorable survival in lung adenocarcinoma. Tumour Biol 2013; 34: 2397-2401. https:// doi.org/10.1007/s13277-013-0789-9

[12] HAHN P, WEGENER I, BURRELLS A, BOSE J, WOLF A et al. Analysis of Jmjd6 cellular localization and testing for its involvement in histone demethylation. PLoS One 2010; 5: e13769. https://doi.org/10.1371/journal.pone.0013769

[13] BOECKEL JN, GUARANI V, KOYANAGI M, ROEXE T, LENGELING A et al. Jumonji domain-containing pro- tein 6 (Jmjd6) is required for angiogenic sprouting and regulates splicing of VEGF-receptor 1. Proc Natl Acad Sci U S A 2011; 108: 3276-3281. https://doi.org/10.1073/ pnas. 1008098108

[14] KILLELA PJ, REITMAN ZJ, JIAO Y, BETTEGOWDA $\mathrm{C}$, AGRAWAL $\mathrm{N}$ et al. TERT promoter mutations occur frequently in gliomas and a subset of tumors derived from cells with low rates of self-renewal. Proc Natl Acad Sci U S A 2013; 110: 6021-6026. https://doi.org/10.1073/ pnas. 1303607110

[15] O'KANE R, MATHEW R, KENNY T, STILLER C, CHUMAS P. United Kingdom 30-day mortality rates after surgery for pediatric central nervous system tumors. J Neurosurg Pediatr 2013; 12: 227-234. https://doi. org/10.3171/2013.5.PEDS12514

[16] ZHOU YF, WANG QX, ZHOU HY, CHEN G. Autophagy activation prevents sevoflurane-induced neurotoxicity in H4 human neuroglioma cells. Acta Pharmacol Sin 2016; 37: 580-588. https://doi.org/10.1038/aps.2016.6

[17] HEGI ME, LIU L, HERMAN JG, STUPP R, WICK W et al. Correlation of O6-methylguanine methyltransferase (MGMT) promoter methylation with clinical outcomes in glioblastoma and clinical strategies to modulate MGMT activity. J Clin Oncol 2008; 26: 4189-4199. https://doi. org/10.1200/JCO.2007.11.5964

[18] WELLER M, STUPP R, REIFENBERGER G, BRANDES AA, VAN DEN BENT MJ et al. MGMT promoter methylation in malignant gliomas: ready for personalized medicine? Nat Rev Neurol 2010; 6: 39-51. https://doi.org/10.1038/nrneurol.2009.197

[19] ZIEGLER DS, KUNG AL, KIERAN MW. Anti-apoptosis mechanisms in malignant gliomas. J Clin Oncol 2008; 26: 493-500. https://doi.org/10.1200/JCO.2007.13.9717

[20] FAN X, SALFORD LG, WIDEGREN B. Glioma stem cells: evidence and limitation. Semin Cancer Biol 2007; 17: 214-218. https://doi.org/10.1016/j.semcancer.2006.04.002

[21] FINE HA. Glioma stem cells: not all created equal. Cancer Cell 2009; 15: 247-249. https://doi.org/10.1016/j. ccr.2009.03.010

[22] SINGH SK, HAWKINS C, CLARKE ID, SQUIRE JA, BAYANI $J$ et al. Identification of human brain tumour initiating cells. Nature 2004; 432: 396-401. https://doi.org/10.1038/nature03128

[23] YUAN X, CURTIN J, XIONG Y, LIU G, WASCHSMANNHOGIU $S$ et al. Isolation of cancer stem cells from adult glioblastoma multiforme. Oncogene 2004; 23: 9392-9400. https://doi.org/10.1038/sj.onc.1208311

[24] SINGH SK, CLARKE ID, TERASAKI M, BONN VE, HAWKINS C et al. Identification of a cancer stem cell in human brain tumors. Cancer Res 2003; 63: 5821-5828.

[25] IGNATOVA TN, KUKEKOV VG, LAYWELL ED, SUSLOV ON, VRIONIS FD et al. Human cortical glial tumors contain neural stem-like cells expressing astroglial and neuronal markers in vitro. Glia 2002; 39: 193-206. https://doi.org/10.1002/ glia.10094

[26] GALLI R, BINDA E, ORFANELLI U, CIPELLETTI B, GRITTI A et al. Isolation and characterization of tumorigenic, stem- 
like neural precursors from human glioblastoma. Cancer Res 2004; 64: 7011-7021. https://doi.org/10.1158/0008-5472. CAN-04-1364

[27] HONG X, ZANG J, WHITE J, WANG C, PAN CH et al. Interaction of JMJD6 with single-stranded RNA. Proc Natl Acad Sci U S A 2010; 107: 14568-14572. https://doi.org/10.1073/ pnas. 1008832107

[28] POULARD C, RAMBAUD J, LAVERGNE E, JACQUEMETTON J, RENOIR JM et al. Role of JMJD6 in Breast Tumourigenesis. PLoS One 2015; 10: e0126181. https://doi. org/10.1371/journal.pone.0126181

[29] FENG Z, LEVINE AJ. The regulation of energy metabolism and the IGF-1/mTOR pathways by the $\mathrm{p} 53$ protein. Trends Cell Biol 2010; 20: 427-434. https://doi.org/10.1016/j. tcb.2010.03.004

[30] WANG F, HE L, HUANGYANG P, LIANG J, SI W et al. JMJD6 promotes colon carcinogenesis through negative regulation of p53 by hydroxylation. PLoS Biol 2014; 12: e1001819. https:// doi.org/10.1371/journal.pbio.1001819
[31] ZENG R, HUANG J, ZHONG MZ, LI L, YANG G et al. Multiple Roles of WNT5A in Breast Cancer. Med Sci Monit 2016; 22: 5058-5067. https://doi.org/10.12659/MSM.902022

[32] LIU F, VAN DEN BROEK O, DESTREE O, HOPPLER S. Distinct roles for Xenopus Tcf/Lef genes in mediating specific responses to Wnt/beta-catenin signalling in mesoderm development. Development 2005; 132: 5375-5385. https:// doi.org/10.1242/dev.02152

[33] ZHANG X, GAO Y, LU L, ZHANG Z, GAN S et al. JmjC Domain-containing Protein 6 (Jmjd6) Derepresses the Transcriptional Repressor Transcription Factor 7-like 1 (Tcf7l1) and Is Required for Body Axis Patterning during Xenopus Embryogenesis. J Biol Chem 2015; 290: 20273-20283. https:// doi.org/10.1074/jbc.M115.646554

[34] VAN DE WETERING M1, SANCHO E, VERWEIJ C, DE LAU W, OVING I et al. The beta-catenin/TCF-4 complex imposes a crypt progenitor phenotype on colorectal cancer cells. Cell 2002; 111:241-250. https://doi.org/10.1016/S0092$\underline{\text { 8674(02)01014-0 }}$ 